\title{
Demand-driven Cache Allocation Based on Context-aware Collaborative Filtering
}

\author{
Muhao Chen* \\ Dept. of Computer Science, UCLA \\ muhaochen@cs.ucla.edu
}

\author{
Qi Zhao* \\ Dept. of Computer Science, UCLA \\ qi.zhao@cs.ucla.edu
}

\author{
Pengyuan Du \\ Dept. of Computer Science, UCLA \\ pengyuandu@cs.ucla.edu
}

\author{
Carlo Zaniolo \\ Dept. of Computer Science, UCLA \\ zaniolo@cs.ucla.edu
}

\author{
Mario Gerla \\ Dept. of Computer Science, UCLA \\ gerla@cs.ucla.edu
}

\begin{abstract}
Many recent advances of network caching focus on i) more effectively modeling the preferences of a regional user group to different web contents, and ii) reducing the cost of content delivery by storing the most popular contents in regional caches. However, the context under which the users interact with the network system usually causes tremendous variations in a user group's preferences on the contents. To effectively leverage such contextual information for more efficient network caching, we propose a novel mechanism to incorporate context-aware collaborative filtering into demanddriven caching. By differentiating the characterization of user interests based on a priori contexts, our approach seeks to enhance the cache performance with a more dynamic and fine-grained cache allocation process. In particular, our approach is general and adapts to various types of context information. Our evaluation shows that this new approach significantly outperforms previous non-demanddriven caching strategies by offering much higher cached content rate, especially when utilizing the contextual information.
\end{abstract}

\section{CCS CONCEPTS}

- Network algorithms $\rightarrow$ Embedded systems;

\section{KEYWORDS}

Demand-driven caching, cache allocation, collaborative filtering

\section{INTRODUCTION}

Regional network caches are critical to efficient distribution of web contents in wireless networks, which have attracted much attention recently. Caching highly-demanded contents at wireless edges, which are close to the end users, offers enhanced Quality of Experience (QoE) and much smaller footprints of bandwidth-demanding traffic [?]. Due to the growth of mobile wireless network systems and frequent updating of web contents on servers, there is clearly a

\footnotetext{
*Both authors contribute equally to this work.

Permission to make digital or hard copies of all or part of this work for personal or classroom use is granted without fee provided that copies are not made or distributed for profit or commercial advantage and that copies bear this notice and the full citation on the first page. Copyrights for components of this work owned by others than ACM must be honored. Abstracting with credit is permitted. To copy otherwise, or republish, to post on servers or to redistribute to lists, requires prior specific permission and/or a fee. Request permissions from permissions@acm.org.

Mobihoc '18, June 26-29, 2018, Los Angeles, CA, USA

(C) 2018 Association for Computing Machinery.

ACM ISBN 978-1-4503-5770-8/18/06 . \$ \$15.00

https://doi.org/10.1145/3209582.3225198
}

pressing need of more dynamic and demand-driven approaches for adjusting the cache allocation schema to the evolving user demands. Recent works [? ? ] have adopted collaborative filtering to capture the preferences of content acquisition for regional user groups, based on what highly demanded web contents can be privileged for caching. However, improvements are needed on the following fronts: i) previous approaches require specific contents to be explicitly provided with user ratings, but do not capture the user demands on contents that are reflected by the intensity of content delivery among content providers and regional user groups [? ? ]; ii) no optimized policy is provided to allocate regional caches to serve web content providers by giving fair consideration to content demands of all users in a regional group; iii) the important contextual information that causes the user demands to noticeably vary, such as time and content types, is not modeled by existing approaches.

To address the issues, we propose a novel context-aware cache allocation approach that is driven by the evolving user demands. Our approach leverages the context-aware collaborative filtering (CCF) [?] to learn the latent representations that characterize the user demands on different content providers. In particular, our approach considers the contextual information to differentiate such characterization process, which seeks to enhance the cache efficiency with dynamic and fine-grain adjustment of cache allocation. From our preliminary simulation results, we show the benefit of overall higher cached content rate than existing cache selection strategies by adopting our approach.

\section{CONTEXT-AWARE DEMAND CACHING}

We hereby begin introducing our approach with the notations. We model the network as a bipartite graph $G=(S, U, P)$ where $S$ and $U$ denote content providers and regional user groups respectively, and $P$ denotes the routes between a content provider and a usergroup. The content delivery in $G$ is processed by responding the content requests from a user group $u$, where each request obtains a unit-sized content block either from the regional cache owned by $u$, or directly from the content provider $s$. We use $C$ to denote a set of contexts that differentiate the user demands, then the cache allocation is a distribution function $\Phi_{u}: S \times C \rightarrow[0,1]$ that assigns proportions of a regional cache for the user group $u \in U$ to each content provider $s$, which is constrained by $\sum_{s \in S} \Phi_{u}(s, c)=1$. The content requests in $G$ are aggregated as a 3-dimensional tensor $R_{C} \in \mathbb{R}^{|S| \times|U| \times|C|}$ where each individual entry $r_{s, u, c}$ measures the total blocks of contents that are requested by the user group $u$ from the content provider $s$ conditioned on the context $c$. Assuming $R_{C}$ 
is constructed with abundant history records of content requests, the goal of our approach is to optimize the cache allocation $\Phi_{u}$ based on the user demands on contents that are characterized from $R_{C}$, so as to alleviate the cost for future content delivery in $G$.

\subsection{Context-aware Collaborative Filtering}

2.1.1 Learning Objective. CCF models a decision tensor $F_{C}$ which is decomposed into three embedding matrices $\mathrm{S} \in \mathbb{R}^{|S| \times d}, \mathrm{U} \in$ $\mathbb{R}^{|U| \times d}$ and $\mathrm{C} \in \mathbb{R}^{|C| \times d}(d<\min (|S|,|U|))$ for content providers, regional user groups and contexts respectively, and a central tensor $\mathbf{H} \in \mathbb{R}^{|S| \times|U| \times|C|}$.

$$
F_{C}=\mathbf{H} \times{ }_{1} \mathrm{~S}^{\top} \times \times_{2} \mathrm{U}^{\top} \times{ }_{3} \mathrm{C}^{\top}
$$

$\times_{n}$ thereof is the mode- $n$ tensor product [?]. We use bold-faced $s_{i}$, $\mathbf{u}_{i}, \mathbf{c}_{i}$ to denote the $\mathrm{i}^{t h}$ rows of $S, U, C$, which are the embeddings of content provider $s_{i}$, user group $u_{i}$ and context $c_{i}$ respectively. For simplicity, we fix the central tensor $\mathrm{H}$ as a unit tensor.

The learning objective of CCF is to minimize the following Frobenius loss using optimization algorithms such as SGD [? ],

$$
L=\left\|F_{C}-R_{C}\right\|_{F}=\sqrt{\sum_{s \in S, u \in U, c \in C} \operatorname{dis}\left(f_{s, u, c}, r_{s, u, c}\right)}
$$

for which the dissimilarity function dis is defined as

$$
\operatorname{dis}(f, r)=\left\{\begin{array}{ll}
(f-r)^{2} & \text { if } r>0 \\
0 & \text { if } r=0
\end{array} \quad\right. \text { (indicating no record) }
$$

A well-trained CCF model produces the embedding representations of content providers and user groups under specific contexts with powerful similarity inferences based on their Euclidean distances. More importantly, $f_{s_{i}, u_{j}, c_{k}}=\left(\mathbf{s}_{i} \odot \mathbf{u}_{j}\right) \cdot \mathbf{c}_{k}$ predicts the demand of user group $u_{i}$ on content provider $s_{j}$ under context $c_{k}$.

2.1.2 Contextual Information. There are multiple categories of contextual information to be adopted to differentiate the user demands, for example:

- Time: $C$ may adopt time information to capture the differences of user demands in temporal contexts. For examples, users in residential areas may expect more cache allocation for video streaming servers in weekends than in weekdays.

- Content types: the user demand on contents from one content provider may also vary largely for different content types such as text, multimedia and structured data.

It is noteworthy that such context should not overlap with the distinctive features of regional caches such as locations, which is likely to cause significant bias in the CCF learning process.

2.1.3 Aggregation of Content Requests. We aggregate $r_{s, u, c}$ based on the number of content blocks that user group $u$ has acquired from content provider $s$ under context $c$, then each entry $r_{s, u, c}$ is divided by the maximum value of $R_{C}$ for normalization.

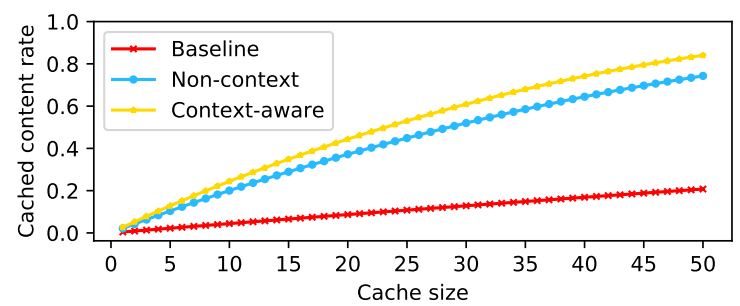

Figure 1: Cached content rates for: 1) context-aware demanddriven (proposed), 2) non-context-aware demand-driven, and 3) non-demand-driven caching.

\subsection{Demand-driven Regional Cache Allocation}

Once the CCF model obtains the embedding representations, then the cache allocation function $\Phi_{u}$ that decides the proportion of the regional cache dedicated to content provider $s_{i}$ under context $c$, can be modeled as a softmax:

$$
\Phi_{u}\left(s_{i}, c\right)=\frac{\exp \left(\left(\mathbf{s}_{i} \odot \mathbf{u}\right) \cdot \mathbf{c}\right)}{\left.\sum_{s \in S} \exp ((\mathbf{s} \odot \mathbf{u}) \cdot \mathbf{c})\right)}
$$

\subsection{Simulation and Evaluation}

We evaluate based on the metric of cached content rate [? ], i.e., the percentage of contents delivered by caches. Assuming that the number of requested content blocks from each content provider follows a uniform distribution, then the cached content rate is estimated by the proportion of content block requests that are satisfied by the regional caches, which is calculated as below.

$$
\psi=\frac{\sum_{s \in S, u \in U, c \in c} h i t(s, u, c) r_{s, u, c}^{\prime}}{\sum_{s \in S, u \in U, c \in c} r_{s, u, c}^{\prime}}
$$

$r_{s, u, c}^{\prime}$ is the future record of aggregated content blocks delivered from $s$ to $u$ under context $c$ that is unknown to $R_{C}$. The cache hit rate function hit is defined as follows, where $m_{u}$ is the maximum size of the regional cache that user group $u$ has.

$$
h i t(s, u, c)= \begin{cases}\frac{\Phi_{u}(s, c) m_{u}}{r_{s, u, c}^{\prime}} & \text { if } \Phi_{u}(s, c) m_{u}<r_{s, u, c}^{\prime} \\ 1 & \text { if } \Phi_{u}(s, c) m_{u} \geq r_{s, u, c}^{\prime}\end{cases}
$$

We conduct 10-fold cross-validation to evaluate on the simulation data of content requests among 500 user groups and 500 content providers under seven temporal contexts. The results are reported in Fig. 1, which show that the demand-driven caching promisingly increases the proportion of contents to be delivered directly from the cache, and indicate that the overall content delivery cost in the network can be effectively reduced. On top of that, the contentaware strategy further leads to a moderate improvement. 